\title{
B-type natriuretic peptide and renal function in Japanese patients with type 2 diabetes mellitus: The Dogo Study
}

\author{
Shinya Furukawa ${ }^{1), 2)}$, Takenori Sakai ${ }^{3)}$, Tetsuji Niiya ${ }^{4)}$, Hiroaki Miyaoka ${ }^{5)}$, Teruki Miyake ${ }^{6)}$, \\ Shin Yamamoto ${ }^{7)}$, Keiko Tanaka ${ }^{1), 2)}$, Teruhisa Ueda ${ }^{8)}$, Hidenori Senba ${ }^{1), 6)}$, Masamoto Torisu ${ }^{9)}$, \\ Hisaka Minami $^{10)}$, Bunzo Matsuura ${ }^{7)}$, Yoichi Hiasa ${ }^{6)}$ and Yoshihiro Miyake ${ }^{1), 2)}$ \\ 1) Department of Epidemiology and Preventive Medicine, Ehime University Graduate School of Medicine, Toon, Japan \\ 2) Epidemiology and Medical Statistics Unit, Translational Research Center, Ehime University Hospital, Toon, Japan \\ 3) Department of Internal Medicine, Yawatahama City General Hospital, Yawatahama, Japan \\ 4) Department of Internal Medicine, Matsuyama Shimin Hospital, Matsuyama, Japan \\ 5) Department of Internal Medicine, Saiseikai Matsuyama Hospital, Matsuyama, Japan \\ 6) Department of Gastroenterology and Metabology, Ehime University Graduate School of Medicine, Toon, Japan \\ 7) Department of Lifestyle-related Medicine and Endocrinology, Ehime University Graduate School of Medicine, Toon, Japan \\ 8) Department of Internal Medicine, Ehime Prefectural Central Hospital, Matsuyama, Japan \\ 9) Department of Internal Medicine, Saiseikai Saijo Hospital, Saijo, Japan \\ 10) Department of Internal Medicine, Ehime Prefectural Niihama Hospital, Niihama, Japan
}

\begin{abstract}
Among patients with type 2 diabetes mellitus, the association between B-type natriuretic peptide (BNP) and renal function remains controversial. We therefore investigated this issue among Japanese patients with type 2 diabetes mellitus. This study included 687 Japanese patients with type 2 diabetes mellitus. BNP levels were divided at quartile points on the basis of the distribution. We used four outcomes regarding the renal function: 1) chronic kidney disease (CKD): estimated glomerular filtration rate $(\mathrm{eGFR})<60 \mathrm{~mL} / \mathrm{min} / 1.72 \mathrm{~m}^{2}, 2$ ) advanced CKD: eGFR $<30 \mathrm{~mL} / \mathrm{min} / 1.72 \mathrm{~m}^{2}$, 3) microalbuminuria: urinary albumin creatinine ratio (UACR) $\geq 30 \mathrm{mg} / \mathrm{g}$ creatinine, and 4) macroalbuminuria: UACR $\geq$ $300 \mathrm{mg} / \mathrm{g}$ creatinine. The prevalence values of CKD, advanced CKD, microalbuminuria, and macroalbuminuria were 27.4 , $2.5 \%, 31.4 \%$, and $9.3 \%$, respectively. Highest BNP ( $\geq 39.2 \mathrm{ng} / \mathrm{mL})$ was independently positively associated with microalbuminuria and macroalbuminuria (adjusted ORs, 2.61 [95\% CI: 1.53-4.49] and 3.45 [95\% CI: 1.46-8.72], respectively). High BNP was not associated with advanced CKD or CKD. There was a statistically significant positive exposure-response relationships between the BNP level and advanced CKD, microalbuminuria, and macroalbuminuria ( $\mathrm{p}$ for trend $=0.047,0.001$, and 0.003 , respectively). BNP level may be independently positively associated with advanced CKD, microalbuminuria, and macroalbuminuria but not CKD in Japanese patients with type 2 diabetes mellitus.
\end{abstract}

Key words: Albuminuria, BNP, Chronic kidney disease, Diabetic nephropathy

THE LEADING CAUSE OF THE DEATH among patients with renal dysfunction is cardiovascular diseases [1], and the importance of the interaction between the heart and kidneys is widely note [2]. Plasma B-type natriuretic peptide (BNP) releases from the ventricles in response to hemodynamic stress, and BNP is one of the predictive markers for cardiovascular events $[3,4]$.

Among patients with type 2 diabetes mellitus, sev-

Submitted Jun. 11, 2017; Accepted Jul. 30, 2017 as EJ17-0256 Released online in J-STAGE as advance publication Aug. 31, 2017 Correspondence to: Shinya Furukawa,Department of Epidemiology and Preventive Medicine, Ehime University Graduate School of Medicine, Shitsukawa, Toon, Ehime 791-0295, Japan.

E-mail: shinya.furukawa@gmail.com eral pieces of evidence exist regarding the association between BNP level and renal function. In a Japanese study, BNP levels among diabetic patients with microalbuminuria were higher than in those without [5]. BNP level was significantly positively associated with urinary albumin-creatinine ratio (UACR) in a Japanese study of patients with type 2 diabetes mellitus [6]. In a Japanese study of diabetic patients without coronary heart diseases, BNP level was positively associated with macroalbuminuria but not microalbuminuria [7]. On the other hand, in two Japanese studies of normotensive patients with type 2 diabetes mellitus, BNP level was not associated with either microalbuminuria [8] or proteinuria [9]. The association between BNP 
level and renal function among patients with type 2 diabetes mellitus was still inconclusive. The purpose of this study was to estimate the association between plasma BNP level and renal function among Japanese patients with type 2 diabetes mellitus.

\section{Methods}

\section{Study population}

The Dogo Study is a multicenter prospective cohort study that recruited 1,051 Japanese patients, from September 2009 to September 2014, with previously diagnosed type 2 diabetes mellitus (median age at recruitment, 61.6 years; range, $19-88$ years; $60.9 \%$ men). Collaborating physicians (from 10 hospitals) who specialize in diabetes mellitus were responsible for the diagnosis of type 2 diabetes mellitus, according to the Japan Diabetes Society criteria. There were 364 patients excluded from the current analysis because of incomplete data on the variables under study. Thus, the final analysis sample consisted of 687 patients. The present study protocol received ethics approval from the institutional review board of Ehime University Graduate School of Medicine. Written informed consent was obtained from all patients enrolled in The Dogo Study.

\section{Subjects' characteristics and outcome measures}

Each participant completed a self-administered questionnaire, which collected data on smoking habits, diabetes duration, current drinking habits, use of antihypertensive medication, and use of anti-hyperlipidemic medication. Each patient's body mass index (BMI) was calculated as their weight $(\mathrm{kg})$ divided by the square of their height $\left(\mathrm{m}^{2}\right)$. Blood pressure was measured in the sitting position using a cuff, after a rest period of at least $5 \mathrm{~min}$. A patient was considered to be hypertensive if the systolic blood pressure was $\geq 140$ $\mathrm{mmHg}$, diastolic blood pressure was $\geq 90 \mathrm{mmHg}$, or both. Dyslipidemia was defined as positive if the serum low-density lipoprotein cholesterol concentration was $\geq 140 \mathrm{mg} / \mathrm{dL}$, triglyceride concentration was $\geq 150 \mathrm{mg} /$ $\mathrm{dL}$ or high-density lipoprotein cholesterol concentration was $<40 \mathrm{mg} / \mathrm{dL}$, or if the patients were already being treated with lipid-lowering agents. Stroke and ischemic heart disease were assessed based on the selfadministered questionnaires, medical records, and/or admission data. Use of insulin and/or oral anti-hyperglycemic agents was determined by medical records.
The definition of diabetic retinopathy was based on the Fukuda standard [10]. The definition of diabetic neuropathy was based on the abbreviated diagnosis of diabetic neuropathy [11].

\section{Assessment of B-type natriuretic peptide}

Blood samples were taken the morning after overnight fasting. The plasma BNP level concentration was measured with specific chemiluminescent enzyme immunoassay using a human BNP kit (Shionogi, Osaka, Japan).

\section{Assessment of renal function}

Estimated glomerular filtration rate (eGFR) was calculated using serum creatinine $(\mathrm{Cr}): 194 \times$ serum $\mathrm{Cr}^{-1.094} \times$ age $^{-0.287}($ if female $\times 0.724)$ [12]. The UACR was assessed using urine samples that were taken once in the morning from the first void. We used four outcomes: 1) CKD: eGFR $<60 \mathrm{~mL} / \mathrm{min} / 1.72 \mathrm{~m}^{2}$ (regardless of UACR), 2) advanced CKD: eGFR $<30 \mathrm{~mL} /$ $\mathrm{min} / 1.72 \mathrm{~m}^{2}$ (regardless of UACR), 3) microalbuminuria: UACR $\geq 30 \mathrm{mg} / \mathrm{g}$ creatinine (regardless of eGFR status), and 4) macroalbuminuria: UACR $\geq 300 \mathrm{mg} / \mathrm{g}$ creatinine (regardless of eGFR status).

\section{Statistical analysis}

Subjects were divided into quartiles with the following cut-off points: 1) $\mathrm{BNP}<9.9$ (reference), 2): $9.9 \leq$ $\mathrm{BNP}<19.0$, 3) $19.0 \leq \mathrm{BNP}<39.2$, and 4) $\mathrm{BNP} \geq 39.2$. We selected sex, age, body mass index, duration of type 2 diabetes, current smoking, current drinking, hypertension, dyslipidemia glycated hemoglobin, stroke, ischemic heart disease, and use of pioglitazone as potential confounding factors. Logistic regression analysis was conducted to estimate adjusted odds ratios (ORs) and the $95 \%$ confidence intervals (CI). Multiple regression logistic analyses were used to adjust for potential confounding factors. Trend of an association was assessed using a logistic regression model assigning consecutive integers to the BNP level categories. All analyses were carried out using the SAS software package version 9.4 (SAS Institute, Inc., Cary, NC, USA).

\section{Results}

Characteristics of the 687 patients are listed in Table 1. Of the 687 patients with type 2 diabetes mellitus, mean age, $\mathrm{HbAlc}$, and duration of type 2 diabetes mellitus were 61.9 years old, $7.93 \%$, and 10.7 
Table 1 Clinical characteristics of the 687 study participants

\begin{tabular}{|c|c|}
\hline Variable & $\mathrm{N}(\%)$ \\
\hline Age, years, mean \pm SD & $61.9 \pm 11.2$ \\
\hline Male, $\%$ & $428(62.3)$ \\
\hline BMI, $\mathrm{kg} / \mathrm{m}^{2}$, mean $\pm \mathrm{SD}$ & $25.1 \pm 4.8$ \\
\hline Waist, cm & $87.2 \pm 17.1$ \\
\hline $\mathrm{HbA1c}$, mean $\pm \mathrm{SD}$ & $7.93 \pm 1.87$ \\
\hline Duration of T2DM, years, mean \pm SD & $10.7 \pm 10.2$ \\
\hline Current drinking $(\%)$ & $279(40.6)$ \\
\hline Current smoking (\%) & $124(18.1)$ \\
\hline Hypertension (\%) & $480(69.9)$ \\
\hline Dyslipidemia (\%) & $519(75.6)$ \\
\hline Diabetic retinopathy $(\%)$ & $193(28.1)$ \\
\hline Diabetic neuropathy (\%) & $422(61.4)$ \\
\hline Stroke $(\%)$ & $49(7.1)$ \\
\hline Coronary artery disease $(\%)$ & $79(11.5)$ \\
\hline Use of pioglitazone & $144(21.0)$ \\
\hline UACR & $14.0[1.0-6750.0]$ \\
\hline Microalbuminuria (<30 mg/g Cr) & $216(31.4)$ \\
\hline Macroalbuminuria ( $\leq 300 \mathrm{mg} / \mathrm{g} \mathrm{Cr}$ ) & $64(9.3)$ \\
\hline eGFR & $72.7 \pm 24.7$ \\
\hline $\mathrm{CKD}\left(<60 \mathrm{~mL} / \mathrm{min} / 1.73 \mathrm{~m}^{2}\right)$ & $188(27.4)$ \\
\hline Advanced CKD $\left(<30 \mathrm{~mL} / \mathrm{min} / 1.73 \mathrm{~m}^{2}\right)$ & $17(2.5)$ \\
\hline BNP, $n g / m L$ & $19.0[1.1-898.0]$ \\
\hline$\leq \mathrm{BNP}<9.9$ & $171(24.9)$ \\
\hline $9.9 \leq \mathrm{BNP}<19.0$ & $172(25.0)$ \\
\hline $19.0 \leq \mathrm{BNP}<39.2$ & $169(24.6)$ \\
\hline $39.2 \leq \mathrm{BNP}$ & $175(24.5)$ \\
\hline
\end{tabular}

SD, standard deviation; BMI, body mass index; Hb1 Ac, glycated hemoglobin; T2DM, type 2 diabetes mellitus; UACR, urinary albumin: creatinine ratio; eGFR, estimated glomerular filtration rate; $\mathrm{CKD}$, chronic kidney disease; Cr, creatinine; BNP, B-type natriuretic peptide.

years, respectively. The prevalence values of CKD, advanced CKD, microalbuminuria, and macroalbuminuria were $27.4 \%, 2.5 \%, 31.4 \%$ and $9.3 \%$, respectively. The median BNP level was $19.0 \mathrm{ng} / \mathrm{mL}$. The associations between renal function and BNP level are shown in Table 2. In the crude analysis, compared with BNP levels in the first quartile, BNP levels in the fourth quartile (BNP $\geq 39.2$ ) were positively associated with $\mathrm{CKD}$, advanced CKD, microalbuminuria, and macroalbuminuria. After adjustment for sex, age, body mass index, duration of type 2 diabetes, current smoking, current drinking, hypertension, dyslipidemia, glycated hemoglobin, stroke, ischemic heart disease, and use of pioglitazone, BNP levels in the fourth quartile (BNP $\geq 39.2$ ) were independently positively associated with microalbuminuria and macroalbuminuria: the adjusted ORs were 2.61 (95\% CI: 1.53-4.49) and 3.45 (95\% CI:
1.46-8.72), respectively; however, the positive association between BNP and CKD or advanced CKD had disappeared. Also, there were statistically significant positive exposure-response relationships between the BNP level and advanced CKD, microalbuminuria, and macroalbuminuria ( $\mathrm{p}$ for trend $=0.047,0.001$ and 0.003 , respectively).

\section{Discussion}

The present study demonstrated that plasma BNP level was independently positively associated with advanced $\mathrm{CKD}$, microalbuminuria, and macroalbuminuria among Japanese patients with type 2 diabetes mellitus.

Several pieces of evidence exist regarding the association between BNP and renal function among patients with type 2 diabetes mellitus. In a Japanese study of normotensive, lean patients with type 2 diabetes mellitus, BNP level was positively associated with UACR [5]. A positive association between BNP level and UACR was found in a Japanese study of 316 patients with type 2 diabetes mellitus [6]. In a Japanese study of 233 patients with type 2 diabetes mellitus, BNP level was positively associated with macroalbuminuria [7]. These findings are in partial agreement with our results. On the other hand, in a Japanese study of 100 normotensive patients with type 2 diabetes mellitus, BNP level was not positively associated with microalbuminuria [8]. In another Japanese study of 100 normotensive patients with type 2 diabetes mellitus, BNP level was not associated with proteinuria [9]. In a Japanese cohort study of 97 patients with type 2 diabetes mellitus and microalbuminuria, low BNP at baseline was a predictor for remission of diabetic nephropathy [13].

The median BNP and the mean BNP among patients in the present study were $19.0 \mathrm{ng} / \mathrm{mL}$ and $42.5 \mathrm{ng} / \mathrm{mL}$ (data not shown), respectively. In previous epidemiological studies regarding the association BNP and renal function, plasma BNP ranged from 10.8 to $46.3 \mathrm{ng} / \mathrm{dL}$ [5-9]. The discrepancies among these studies may be explained, at least in part, by differences in sample size; the definition of renal function; characteristics such as age, sex, race, BMI, prevalence of coronary heart diseases, subclinical heart failure and hypertension; and other confounding factors.

In two studies of patients with type 2 diabetes mellitus, eGFR was independently positively associated with onset of CVD among patients with albuminuria but not among those without albuminuria $[14,15]$. In 
Table 2 Crude and adjusted odds ratios and 95\% confidence intervals for renal dysfunction in relation to plasma level of BNP

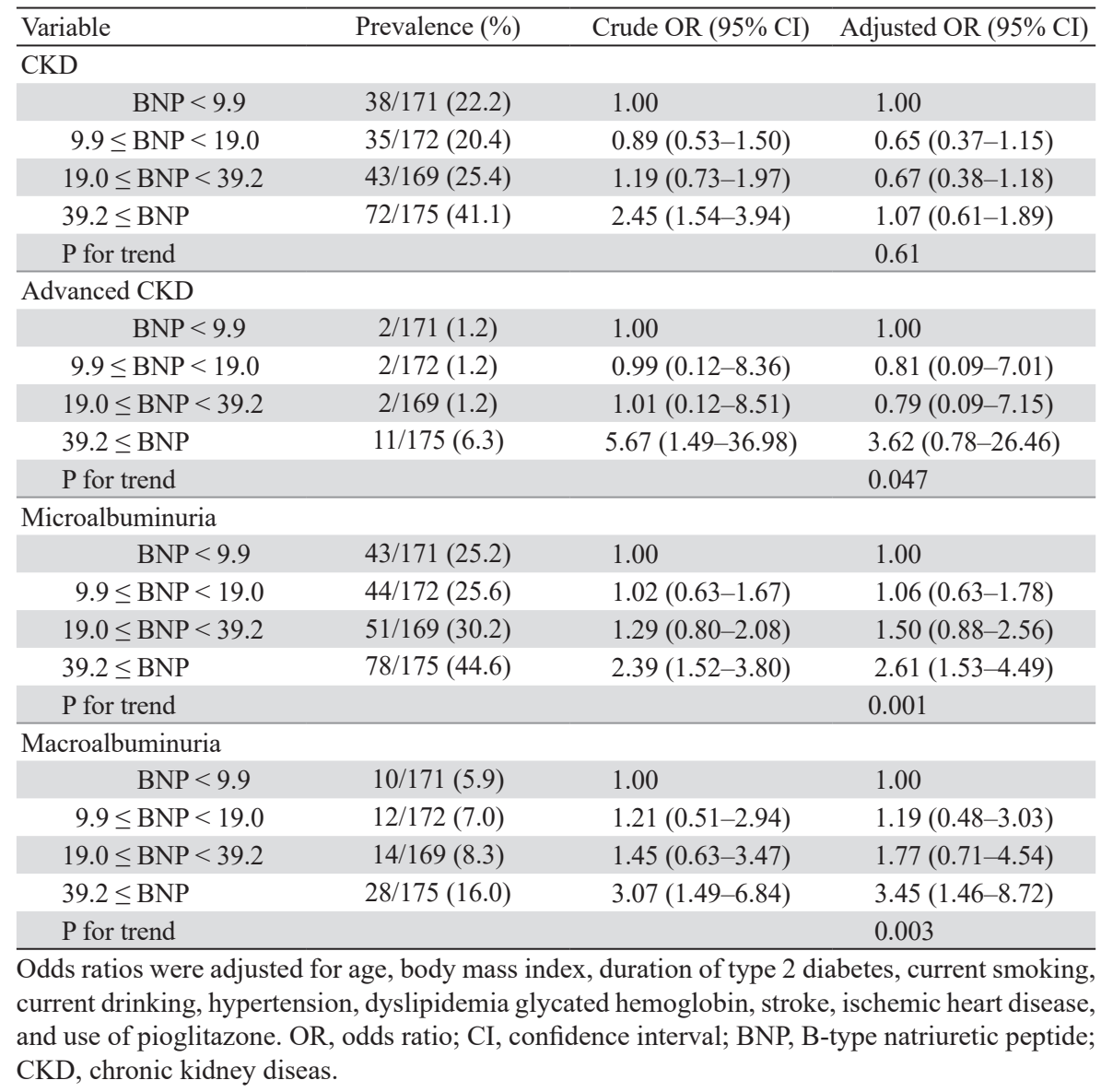

a previous study of patients with type 2 diabetes mellitus, advanced CKD but not mild to moderate CKD was significantly positively associated with CVD, regardless of albuminuria status [16]. In the present study, plasma BNP was independently positively associated with macroalbuminuria, regardless of eGFR. The association between plasma BNP level and advanced CKD was marginally significant. In contrast, plasma BNP was not associated with prevalence of CKD. Our results in the present study were consistent with the findings in previous studies that showed the association between renal function and onset of CVD among patients with type 2 diabetes mellitus.

In clinical practice, decreasing plasma BNP might be a useful marker for remission of albuminuria but not eGFR. However, further research regarding the association between BNP and renal function is needed to assess the usefulness of BNP as a prognostic marker for albuminuria among patients with type 2 diabetes mellitus.
Cardio-renal syndrome has received widespread attention [2]. However, the mechanism of the association between BNP and albuminuria among patients with type 2 diabetes mellitus is still not clear. In patients with heart failure, deceased clearance from the kidneys contributes to the elevated BNP level [17]. Among patients with type 1 diabetes mellitus, BNP infusion increased urine excretion of albumin [18]. Low clearance of BNP might therefore increase urine excretion of albumin.

Several limitations of the present study warrant mention. First, this study was cross-sectional by design. Second, we measured the BNP level, eGFR, and UACR only once. Third, we could not exclude asymptomatic heart disease including heart failure, valvular disease, arrhythmia, and cardiomyopathy. Fourth, selection bias should have influenced our results. The subjects in the present study were therefore likely not representative of Japanese patients with type 2 diabetes mellitus. 
However, in a Japanese community-based diabetes study of 1059 patients with diabetes [19], the middle quintiles of BNP (14.5-16.3 ng/dL) were similar to the results in our study $(19.0 \mathrm{ng} / \mathrm{dL})$. According to the Japanese Diabetes Clinical Management (JDDM) Study, the prevalence values of microalbuminuria and macroalbuminuria (31.6\% and $10.5 \%$, respectively) [20] were similar to those in the present study (31.4\% and $9.3 \%$, respectively). Fifth, sample size in the present study might not be large enough to clearly show the association between high BNP and advanced CKD among patients with type 2 diabetes mellitus. Finally, most of the patients in this cohort may have been receiving treatment for a considerable period of time. Physicians may have opted to attempt to control high BNP levels and protect renal function. The long duration of such treatment might have masked the association between BNP level and renal function.
In conclusion, BNP level may be independently positively associated with advanced CKD, microalbuminuria, and macroalbuminuria but not CKD in Japanese patients with type 2 diabetes mellitus.

\section{Acknowledgements}

The authors would thank The Dogo Study group: Morikazu Onji from Saiseikai Imabari Hospital, Keiko Kikuchi and Tomo Kogama from Ehime University, and Eri Furukawa from the Furukawa Clinic. The Dogo Study was supported by the Japan Society for the Promotion of Science (JSPS) KAKENHI Grants (21790583 and 23790697).

\section{Disclosure}

The authors declare no conflicts of interest.

\section{References}

1. Parfrey PS, Foley RN (1999) The clinical epidemiology of cardiac disease in chronic renal failure. J Am Soc Nephrol 10: 1606-1615.

2. Ronco C, Haapio M, House AA, Anavekar N, Bellomo R (2008) Cario-renal syndrome. J Am Coll Cardiol 52: 1527-1539.

3. Onodera M, Nakamura M, Tanaka F, Takahashi T, Makita S, et al. (2012) Plasma B-type natriuretic peptide is useful for cardiovascular risk assessment in community-based diabetes subjects. Int Heart J 53: 176-181.

4. Wang TJ, Larson MG, Levy D, Benjamin EJ, Leip EP, et al. (2004) Plasma natriuretic peptide levels and the risk of cardiovascular events and death. $N$ Eng $J$ Med 350: 655-663.

5. Yano Y, Katsuki A, Gabazza EC, Ito K, Fujii M, et al. (1999) Plasma brain natriuretic peptide levels in normotensive noninsulin dependent diabetic patients with microalbuminuria. J Clin Endocrinol Metab 84: 2353-2356.

6. Tanaka Y, Daida H, Imai Y, Miyauchi K, Sato Y, et al. (2009) Morning home blood pressure may be a significant marker of nephropathy in Japanese patients with type 2 diabetes: ADVANCED-J study 1. Hypertens Res 32: 770-774.

7. Igarashi M, Jimbu Y, Hirata A, Tominaga M (2005) Characterization of plasma natriuretic peptide level in patients with type 2 diabetes. Endocr J 52: 353-362.

8. Asakawa H, Fukui T, Tokunaga K, Kawakami F (2002) Plasma brain natriuretic peptide levels in normotensive Type 2 diabetic patients without cardiac disease and mac- roalbuminuria. J Diabetes Complications 16: 209-213.

9. Isotani H, Kameoka K, Sasaki I, Hida H, Kakutani S, et al. (2000) Plasma brain natriuretic peptide levels in normotensive type 2 diabetic patients without cardiac disease. Diabetes Care 23: 859-860.

10. Fukuda M (1994) Classification and treatment of diabetic retinopathy. Diabetes Res Clin Pract 24: S171-176.

11 Yasuda H, Sanada M, Kitada K, Terashima T, Kim H, et al. (2007) Rationale and usefulness of newly devised abbreviated diagnostic criteria and staging for diabetic polyneuropathy. Diabetes Res Clin Pract 77: S178-183.

12. Matsuo S, Imai E, Horio M, Yasuda Y, Tomita K, et al. (2009) Revised equations for estimated GFR from serum creatinine in Japan Am J Kidney Dis 53: 982-992.

13. Seki N, Nishimura M, Matsumoto T, Fukazawa M, Kenmochi T (2013) Relationship between BNP level and renal function in diabetic nephropathy with microalbuminuria. J Diabetes Complications 27: 92-97.

14. Bouchi R, Bazazono T, Nyumura I, Toya K, Hayashi T, et al. (2009) Is a reduced estimated glomerular filtration rate a risk factor for stroke in patients with type 2 diabetes mellitus. Hypertens Res 32: 381-386.

15. Ninomiya T, Perkovic V, de Galan BE, Zoungas S, Pillai A, et al. (2009) Albuminuria and kidney function independently predict cardiovascular and renal outcomes in diabetes. J Am Soc Nephrol 20: 1813-1821.

16. Bruno G, Merletti F, Bargero G, Novelli G, Melis D, et al. (2007) Estimated glomerular filtration rate, albuminuria and mortality in type 2 diabetes: the Casale 
Monferrato study. Diabetologia 50: 941-948.

17. Tsutamoto T, Wada A, Sakai H, Ishikawa C, Tanaka T, et al. (2006) Relationship between renal function and plasma brain natriuretic peptide in patients with heart failure. J Am Coll Cardiol 47: 582-586.

18. Mckenna K, Smith D. Moore K, Glen A, Tormey W, et al. (2001) Brain natriuretic peptide increases urinary albmin and alpha-1 microglobukin excretion in type 1 diabetes mellitus. Diabet Med 18: 973-978.

19. Onodera M, Kawamura M, Tanaka F, Takahashi T,
Makita S, et al. (2012) Plasma B-type natriuretic peptide is useful for cardiovascular risk assessment in community-based diabetes subjects comparison with albuminuria. Int Heart J 53: 176-181.

20. Yokoyama H, Kawai K, Kobayashi M; Japan Diabetes Clinical Data Management Study Group (2007) Microalbuminuria in common in Japanese type 2 diabetic patients: a nationwide survey from the Japan Diabetes Clinical Data Management Study Group (JDDM 10). Diabetes Care 30: 989-992. 